\title{
Industrial Forum - Industrieforum
}

Fludara ${ }^{\circledR}$ - ein neues Zytostatikum zur Behandlung der chronischen lymphatischen Leukämie (CLL) vom B-Zell-Typ

Zum 1. April 1997 stellt medac, Hamburg, in Kooperation mit Schering, Berlin, das Purinanalogon Fludara ${ }^{\circledR}$ mit dem neuen Wirkstoff Fludarabin-phosphat in Form eines sterilen Lyophilisats zur Verfügung. Fludara ${ }^{\circledR}$ ist zur Therapie der chronischen lymphatischen Leukämie vom B-Zell-Typ (B-CLL) beí Patienten zugelassen, die auf eine Behandlung mit mindestens einem - eine alkylierende Substanz enthaltenden - Standard-Therapieschema nicht ansprachen oder deren Krankheit während oder nach einer derartigen Behandlung fortschritt. Nach aseptischer Rekon-stitution wird die neue Zubereitung dem Patienten entweder als Bolusinjektion oder mit Kochsalzlösung verdünnt über eine Dauer von etwa 30 Minuten intravenös verabreicht.

Die positiven Ergebnisse vieler klinischer Studien belegen den hohen Nutzen, den Patienten àus diesem wichtigen Fortschritt in der Therapie der B-CLL ziehen: Es werden hohe Gesamtremissionsraten mit einem signifikanten Anteil an Komplett-Remissionen erzielt. Mit Fludara ${ }^{\circledR}$, das erstmals in Deutschland von medac, Hamburg, in den Handel gebracht wird, wird der deutsche Arzneimittelmarkt um ein neues Therapeutikum zur Behandlung der B-CLL bereichert, das sich durch eine hohe Wirksamkeit bei niedriger therapieassoziierter Toxizität auszeichnet.

medac

Gesellschaft für klinische Spezialpräparate $\mathrm{mbH}$

Dr. Erich Enghofer

Fehlandtstraße 3

20354 Hamburg

Tel. 040/35091-0

Neueinführung Fluta-GRY®

Zum 1. Februar hat GRY-Pharma Fluta-GRY® eingeführt. Fluta-GRY® mit dem Wirkstoff Flutamid ist ein Antiandrogen zur hormonellen Therapie des fortgeschrittenen

Prostatakarzinoms. Damit erweitert GRY-Pharma das bestehende Sortiment in der Onkologie um ein weiteres Qualitätsprodukt. Fluta-GRY ${ }^{\circledR}$ wird in einer patientenfreundlichen und compliancefördernden Kalenderpackung angeboten. Der äußerst niedrige Preis ermöglicht eine sichere und ausgesprochen wirtschaftliche Therapie.

C.G. Schmidt-Stipendium erstmals verliehen -Forscherin der Mainzer Universitätsklinik untersucht neuen Therapieansatz bei malignen Melanomen

Die erste Stipendiatin des 1996 ausgeschriebenen C.G. Schmidt-Stipen-diums ist Dr. med. Bianca M. Wittig von der 1. Medizinischen Klinik und Poliklinik der Johannes-GutenbergUniversität Mainz. Sie erhielt die Forschungsförderung am 7. Februar 1997 in Essen.

Das C. G. Schmidt-Stipendium ist mit 30 000,- DM dotiert und wird von dem Erlanger Arzneimittelunternehmen Pharmacia \& Upjohn gestiftet. Vorsitzender des Kuratoriums «CG 
Schmidt-Stipendium» ist Professor Dr. med. Siegfried Seeber, Direktor des Westdeutschen Tumorzentrums, Essen.

Dr. med. Bianca M. Wittig beschreibt in ihrem Forschungsantrag, daß kon-ventionelle Therapieregime nach metastatischer Dissemination bei vielen Tumorarten nur noch palliativ wirksam sind. Auf der Suche nach alterna-tiven Methoden wurde in letzter Zeit eine Reihe von T-Zell-erkannten Antigenen identifiziert und somit Ansätze für tumorimmunologische Behandlungsmethoden bei bestimmten menschlichen Tumoren definiert. Im Rahmen des Stipendiums soil ein Protokoll etabliert werden, das den autologen Transfer von tumorspezifischen zytotoxischen T-Zellen ermöglicht. Das dem Projekt zugrundeliegende Konzept ermöglicht die Unter-suchung weiterer tumorspezifischer oder tumorassoziierter Antigene und somit längerfristig eine breite klinische Anwendung. Im Rahmen der Verleihung des C. G. Schmidt-Stipendiums beschrieb Prof. Dr. med. Siegfried Seeber den Forschungsansatz von Dr. Bianca M. Wittig als innovativ und im Sinne zukünftiger immunologischer Therapieansätze als relevant. Natürlich sind gerade die immuntherapeutischen Aspekte der Tumorbehandlung immer noch sehr begrenzt und nur bei wenigen Tumoren erfolgversprechend, so z. B. beim zu untersuchenden malignen Melanom.

Prof. Siegfried Seeber halt es gerade in Anbetracht der derzeit schwierigen Situation junger Mediziner für besonders anerkennenswert, daß Pharmacia \& Upjohn diese Initiative zur gezielten fachlichen und wissenschaftlichen Weiterbildung ermöglicht hat.

Pharmacia \& Upjohn GmbH

Helmut Schäfers

Hofmannstraße 26

D-91052 Erlangen

Tel. 09131/801-344, Fax 09131/801-345

Neueinführung Vincristinsulfat-GRY® 5

Zum 17. Februar 1997 hat GRY-Pharma Vincristinsulfat-GRY® 5 eingeführt. GRY-Pharma bietet als einziger Hersteller auf dem deutschen Markt das Vincaalkaloid Vincristin in der Stärke $5 \mathrm{mg}$ an. Die Vorzüge dieser Packungsgröße verbinden wichtige Sicherheitsaspekte mit ökonomischen und ökologischen Vorteilen: erhöhte Zytostatikasicherheit bei Bedarf größerer Substanzmengen, z. B. in der zentralen Zytostatikazu-bereitung, geringerer Aufwand und Reduktion der Müllmenge. Die Fertiglösung kann nach Anbruch 14 Tage lang im Kühlschrank aufbewahrt werden. Vincristinsulfat-GRY ${ }^{\circledR} 5$ ergänzt das bestehende Sortiment der qualitativ hochwertigen und preiswerten GRY-Markenzytostatika.

GRY-Pharma GmbH Kandelstraße 10 D-79199 Kirchzarten Tel.: 07661/984501 Fax: 07661/7159

Topotecan - der erste zugelassene Topoisomerase-I-Inhibitor in Deutschland Mit dem Camptothecinderivat Topotecan (Hycamtin $\left.{ }^{\circledR}\right)$ steht jetzt der erste Topoisomerase-IInhibitor zur Behandlung des fortgeschrittenen Ovarial-karzinoms zur Verfügung. Am 17. Februar 1997 wurde Topotecan unter dem Handelsnamen Hycamtin® in den deutschen Markt eingeführt. Die europäische Zulassung für die Indikation «fortgeschrittenes Ovarialkarzinom» hatte die European Medical Evaluation Agency (EMEA) bereits am 12 . November 1996 erteilt. Die empfohlene Dosierung beträgt 1,5 mg/m" Topotecan als 30minütige intravenöse Kurzinfusion, Tage 1-5, alle 3 Wochen. Die gute Aktivität von Topotecan beim fortgeschrittenen Ovarialkarzi-nom wird in verschiedenen Phase-II-Studien und in einer Phase- 
III-Studie belegt. Hervorzuheben ist, daß Topotecan nicht nur bei Platin- und Pacli-taxel-haltiger Vorbehandlung, sondern auch bei Platin- und Paclitaxel-

\section{KÄÏIGER}

(C) 1997 S. Kargcr GmbH, Freiburg Fax (0761) 4520714 http://www.karger.ch

refraktären bzw. -resistenten Patientinnen noch ein Ansprechen indu-

zieren kann. Im Rahmen der Phase-I $\prod-S t u d i e$ gegen Paclitaxel hatte sich

Topotecan als mindestens gleichwertig erwiesen.

Die guten Ergebnisse basieren wesentlich auf einem neuen antineopla-

stischen Wirkprinzip. Topotecan hemmt das Enzym Topoisomerase-I. Das

führt zu einer Störung der DNS-Replikation, in der Folge zu Strang-

brüchen und zum Tod der Zelle.

Topotecan kann außerdem die Blut-Hirn-Schranke durchdringen und ist bei Patienten mit Hirnmetastasen aktiv.

Weitere erfolgversprechende Indikationen sind unter anderem das kleinzellige und das nichtkleinzellige Bronchialkarzinom sowie Hämoblastosen.

SmithKline Beecham Pharma Leopoldstraße 175 D-80809 München

Neueinführung: Aciclovir 200/400 BRAHMS

Aciclovir 200 BRAHMS steht als Nl mit 25 Tabletten zur Verfügung, Aciclovir 400 BRAHMS

in den Packungsgrößen N2 mit 35 Tabletten und N3 mit 70 Tabletten. Mit diesen Präparaten soil

dem Arzt die wirt-schaftliche Verordnung eines bewährten und hochwirksamen Produktes

erleichtert werden.

Die B.R.A.H.M.S Arzneimittel GmbH ist eine Tochtergesellschaft der B.R.A.H.M.S Diagnostica GmbH (1994 aus der Henning Berlin hervorge-gangen), die ihrerseits ein führendes

biotechnologisches Unternehmen ist. Forschungsschwerpunkte sind Infektiologie-Diagnostik und Schilddrüsen-Diagnostik.

Für weitere Fragen steht Ihnen B.R.A.H.M.S Arzneimittel in Wiesbaden gerne zur Verfügung. B.R.A.H.M.S Arzneimittel GmbH Kreuzberger Ring 13 D-65205 Wiesbaden Tel. 0611/97782-0 Fax 0611/9778222

B.R.A.H.M.S Arzneimittel GmbH, Wiesbaden, teilt mit, daß ab sofort das Virustatikum Aciclovir in den Stärken 200 und $400 \mathrm{mg}$ zur Verfügung steht.

ÖHKCL® $\ll \mathrm{iE}$

Fachliteratur, die überzeugt!

\section{AHMAI AfilF}

Informiert über kliniknahe Grundlagenforschung, klinische Krebsforschung und -behandlung

Ich abonniere

die Zeitschrift Onkologieab Jahrgang

o zum regulären Preis von DEM 182.-

a zum Sonderpreis von DEM 91.- für Mitglieder dera AIO ‘ CAO

a DGHO $\propto$ ÖGHO (ausschließlich an Privatadresse)

Diese Preise gelten für 1997 und verstehen sich inkl. MWSt., exkl.Versandkosten.

Preisänderungen vorbehalten.

a Bitte senden Sie mir ein kostenloses Probeheft von Onkologie 
Ich kann diese Bestellung innerhalb einer Woche durch eine einfache Mitt1/8ilung an den Verlag wider-rufen. Das Abonnement gilt zunächst für ein Jahr und verlängert sich um jeweils ein weiteres Jahr, wenn ich die Zeitschrift nicht bis I. Dezember des laufenden Jahres abbestelle.

KAKGEÍl

S. Karger $\mathrm{GmbH}$

Postfach

D-79095 Freiburg

(Deutschland)

http://www.karger.chl

journalslonklonkdes.htm

Ich bezahle a mit Scheck a auf Rechnung

Bitte belasten Sie diese Bestellung meiner Kreditkarte

a American Express a Eurocard

Karten-Nr. Ablauf

Ich ermächtige die S. Karger GmbH bis aufWiderruf, jährlich meine Abonnement-Gebühren im Lastschriftverfahren abzubuchen (nur innerhalb Deutschlands möglich) 